KINDER- UND JUGENDLITERATURFORSCHUNG 2000/2001 


\section{Kinder- und \\ Jugendliteraturforschung 2000/2001}

Mit einer Gesamtbibliographie

der Veröffentlichungen des Jahres 2000

In Zusammenarbeit mit der Gesellschaft für Kinder- und

Jugendliteraturforschung in Deutschland und der deutschsprachigen Schweiz, der Österreichischen Gesellschaft für Kinder- und Jugendliteraturforschung und in Verbindung mit Verena Rutschmann (Zürich),

Ernst Seibert (Wien)und Jack Zipes (Minneapolis)

herausgegeben von

Hans-Heino Ewers, Ulrich Nassen, Carola Pohlmann, Karin Richter und Rüdiger Steinlein

Verlag J. B. Metzler Stuttgart • Weimar 
Redaktion: Bernd Dolle-Weinkauff, Hans-Heino Ewers (verantwortlich) Rezensionen: Ulrich Nassen, Jan Bolczyk

Bibliographie: Karin Laubrecht

Layout: Hongqin Flicker

Der Druck dieses Jahrbuchs wurde durch die Gesellschaft für Kinder- und Jugendliteraturforschung in Deutschland und der deutschsprachigen Schweiz gefördert.

Die Deutsche Bibliothek - CIP-Einheitsaufnahme

Kinder- und Jugendliteraturforschung ....

mit einer Gesamtbibliographie der Veröffentlichungen des Jahres ... I

in Zusammenarbeit mit der Arbeitsgemeinschaft Kinder- und

Jugendliteraturforschung. - Stuttgart ; Weimar : Metzler.

Erscheint jährl. - Aufnahme nach 1994/95(1995)

1994/95 (1995) -

\section{ISBN 978-3-476-01871-7 \\ ISBN 978-3-476-02818-1 (eBook) \\ DOI 10.1007/978-3-476-02818-1}

Dieses Werk einschließlich aller seiner Teile ist urheberrechtlich geschützt. Jede Verwertung außerhalb der engen Grenzen des Urheberrechtsgesetzes ist ohne $\mathrm{Zu}$ stimmung des Verlages unzulässig und strafbar. Das gilt insbesondere für Vervielfältigungen, Übersetzungen, Mikroverfilmungen und die Einspeicherung und Verarbeitung in elektronischen Systemen.

(C) 2001 Springer-Verlag GmbH Deutschland

Ursprünglich erschienen bei J. B. Metzlersche Verlagsbuchhandlung und Carl Ernst Poeschel Verlag GmbH in Stuttgart 2001

www.metzlerverlag.de

info@metzlerverlag.de 


\section{Inhalt}

\section{Berichte}

Verena Rutschmann: Das Johanna Spyri-Archiv im 100. Todesjahr der Autorin von Heidi ...3.-. Hans-Heino Ewers: Ansprache zur Emeritierung von Heinz-Jürgen Kliewer am 8. November 2000 [Auszüge] ...5.-. Otto Brunken: Zum 80. Geburtstag von Theodor Brüggemann ...8

\section{Beiträge}

\section{Ute Dettmar}

Von der Rolle.

Zur Theorie und Praxis des Kinderschauspiels im späten 18. und frühen 19. Jahrhundert.

Elisabeth Stuck

Die Schauspieltruppe im Text.

Komödien für Kinder und für Jugendliche in der Schweizer Literatur des 19. Jahrhunderts

Bettina Kümmerling-Meibauer

Im Dschungel des Texts.

Kiplings Dschungelbücher und das Prinzip der asymmetrischen Intertextualität

Andrea Weinmann

Der Leser als Zuhörer von Geschichten.

Leserentwürfe in der westdeutschen Kinderliteratur der fünfziger und sechziger Jahre

Gunter Reiß

Textintentionalität und Adressatenentwurf.

Aspekte der Rezeptionslenkung in Gudrun Pausewangs Die Not der Familie Caldera

Heinrich Kaulen

Aufwachsen in der Mediengesellschaft.

Leserfiguren und Lektüreprozesse in aktuellen Adoleszenzromanen

Gabriele von Glasenapp

Die Zeitalter werden besichtigt.

Zur Inszenierung von Geschichte in der neueren historischen Kinder- und Jugendliteratur

Ute Frey/Karin Richter

Didaktik der Kinder- und Jugendliteratur, Leseförderung

in der „Mediengesellschaft“ und Entwicklung von Medienkompetenz

\section{Forschungsbericht}

Carola Pohlmann

Bibliographien zur historischen deutschsprachigen Kinder- und Jugendliteratur.

Rezensionen 151

Bibliographie. 


\section{Vorwort}

Dieses Jahrbuch ist von Beginn an von einer Forschungsgesellschaft getragen worden. Deren Mitglieder bilden nicht nur einen festen Abnehmerstamm; sie stellen darüber hinaus den weitaus größten Teil der Autorinnen und Autoren, der Rezensentinnen und Rezensenten. An dieser Unterstützung hat sich nichts geändert - bis auf die Tatsache, daß es sich nunmehr um zwei Forschungsgesellschaften handelt. Beide Gesellschaften, deren Namen auf dem Titelblatt nachzulesen sind, sind aus der ehemaligen ,Arbeitsgemeinschaft für Kinder- und Jugendliteraturforschung" hervorgegangen und tragen nun gemeinsam dieses wissenschaftliche Periodikum. - Eine weitere Neuerung besteht darin, daß Carola Pohlmann in den Kreis der Herausgeber eingetreten ist.

Bis auf die beiden letzten gehen dieses Mal alle Beiträge zurück auf Vorträge, die auf der Jahrestagung 2000 der damaligen „Arbeitsgemeinschaft für Kinder- und Jugendliteraturforschung“ gehalten wurden, die vom 22. bis 24. Juni in Bensberg bei Köln stattfand. Das Thema der Tagung lautete „Der Leser im Text“. Die Vorträge sind für den Druck teilweise erheblich überarbeitet worden.

Wie bereits in den vergangenen Jahren, versendet die Redaktion des Jahrbuchs an alle interessierten Bezieher kostenlos eine für IBM-kompatible PC-Systeme (MS-DOS/Windows) ausgelegte Datenbankversion der bisher veröffentlichten Jahresbibliographien (siehe Gutschein am Ende des Bandes). Da der Umfang der Bibliographie die Kapazitäten eine 1,44“-Diskette sprengt, werden seit der Ausgabe 199/2000 CD-ROM an die Bezieher verteilt.

Anschriften der Herausgeber:

Prof. Dr. Hans-Heino Ewers, Institut für Jugendbuchforschung, der Goethe-Universität, Postfach 111932, 60054 Frankfurt/M.

Prof. Dr. Ulrich Nassen, Institut für Germanistik der Universität, Augustusplatz 9, 04109 Leipzig

Carola Pohlmann, Kinder- und Jugendbuchabteilung der Staatsbibliothek zu Berlin, 10102 Berlin

Prof. Dr. Karin Richter, Pädagogische Hochschule - Institut für Grundschulpädagogik und Kindheitsforschung, 99089 Erfurt

Prof. Dr. Rüdiger Steinlein, Humboldt-Universität, Philosophische Fakultät II, Schützenstr. 21, 10117 Berlin 